\title{
To Study the Helical Antenna for the Biomedical Application
}

\author{
A. K. Jaiswal, Anil kumar, Santosh Tripathi, Amit Tiwari \\ Shiats ,Allahabad, India
}

Obstract: This paper work is a wireless microsensor based dual inductor antenna for sensing the blood activity in artery system. A device that has inductor length is taken 20-mm and diameter of inductor is 3.5-mm and viscosity of blood is vary from $2 * 10^{-3}$ to $4 * 10^{-3}$ (pa.s). The equivalent of inductor coil here as LC tank that will help to find blood pressure and flow rate of the blood in the artery system.

Keywords:Stentenna,MEMS, LC tank

\section{Introduction}

There are mainly two type of blood pressure exist in the body; first one is systolic pressure and second is diastolic pressure. When the heart is being pumped that pressure in arteries is systolic pressure and when the heart is not being pumped that pressure is distolic pressure. [1-2]. During both of these pressure there are two more pressure exist :one is upstream pressure and second one is down stream pressure.

The pressure is changed between two point of LC tank. The change in pressure can be detect by the shift in the frequency at which the external coil shows characteristic in impedence and phase. This paper describes a new dual inductor stentenna[3-5] and its integration of two capacitive pressure sensors to implement a wireless flow sensing system

\section{Method}

When the blood flow through a tank there is a pressure drop between two different location that depend on the flow rate of blood.

For the steady state flow[6].

$\mathrm{P}_{2}-\mathrm{P}_{1}=\mathrm{CV}+\mathrm{DV}^{2}$

Where $\mathrm{P}_{2}$ and $\mathrm{P}_{1}$ are pressure at different location. $\mathrm{V}$ is area averaged flow velocity and $\mathrm{C}$ and $\mathrm{D}$ are constant. The dual inductor design is intended to implement sensing based on this relationship.

If viscosity of blood is viscosity[7],length of tank is L,diameter of arteries is D , and Q is flow rate of blood then by Hagen Poiseuille equation[6]

$\Delta \mathrm{P}=128 \mu \mathrm{LQ} /\left(\pi \mathrm{D}^{4}\right)$

The frequency and amplitude of the longitudinal vibrations of a sensor depends on the length L, elasticity E, and density $\rho$

$$
\mathrm{f}=1 / 2 \mathrm{~L}(\sqrt{\mathrm{E}} / \rho)
$$

The resonance frequency changes when there is a small mass loading on the surface of the sensor. A magnetoelastic sensor of mass $m 0$ with initial resonance frequency $f o$, when subjected to a mass loading of $\Delta m$ demonstrates a decrease in resonance frequency of

$$
\Delta \mathrm{f}=-\mathrm{f}_{0} \Delta \mathrm{m} / 2 \mathrm{~m}_{0}
$$

Further, a change in the viscosity/density of the medium surrounding a sensor creates a damping effect on the sensor vibrations. The shift in resonance frequency $\Delta \mathrm{f}$ is related to the viscosity $\mu$ and density $\rho$ l of the surrounding medium as

$$
\Delta \mathrm{f}=\sqrt{ }\left(\pi \mu \rho \mathrm{f} \mathrm{f}_{0}\right) / 2 \pi \rho_{\mathrm{s}} \mathrm{d}
$$

Where $d$ is the thichness of the magnetoelastic sensor and $\rho_{\mathrm{s}}$ is density.

\section{Equivalent Circuit}

Upan inflation,the deployed stantenna is permanentaly deformed in helical shape, which consist of two coil with 3 turns fig. 1

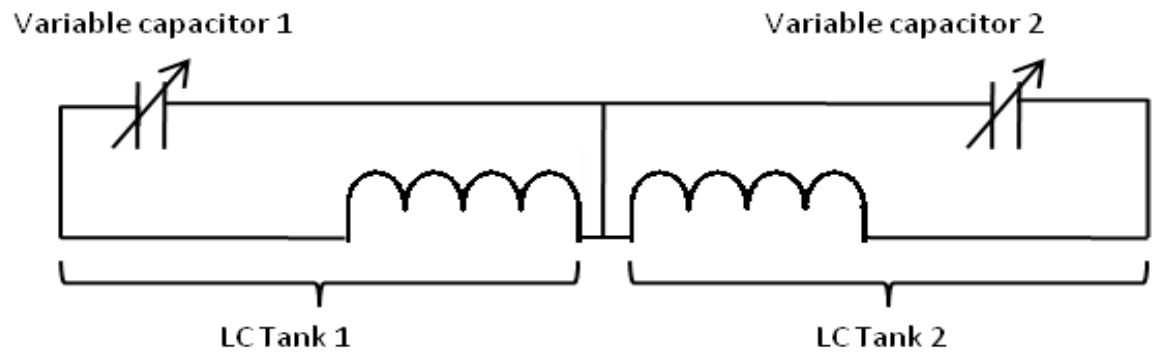

Fig.1 


\section{Result And Discussion}

The motivation for investigating these relationship is to desire too have readily available dialysis access flow estimate for use of each treatment.

It should be recognized that flow monitoring technology will makes frequent and easy measurment throughout each dialysis treatment.

\section{Conclusion}

Fig.2 shows the pressure at upstream and downstream location as a function of flow rate.As can be seen in fig. 2 that pressure response indicate dependence on the flow rate.Its mean that this is a linear graph in which flow rate of blood is vary with variation in pressure linearly.

As in the Fig. 3 shows that the flow rate $\left(\mathrm{mm}^{3} / \mathrm{s}\right)$ of blood is also depends on the viscosity(Pa.s) of the blood.It is clear that flow rate is decrease when the viscosity of blood increase but viscosity of blood is limited to 4 Pa.s.From equation (ii) it is clear that flow rate is propotional to diameter(mm) of arteries but from fig. 4 it is clear that the flow rate is non-linearly vary with diameter.

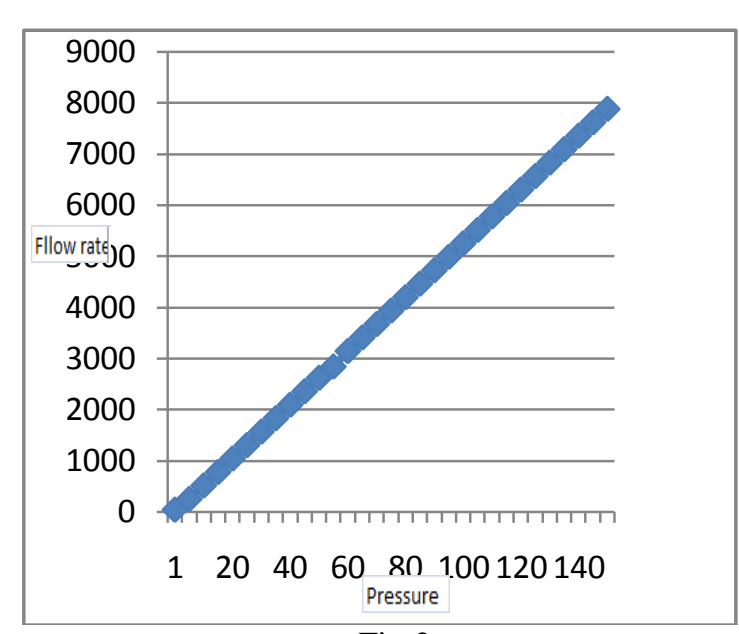

Fig.2

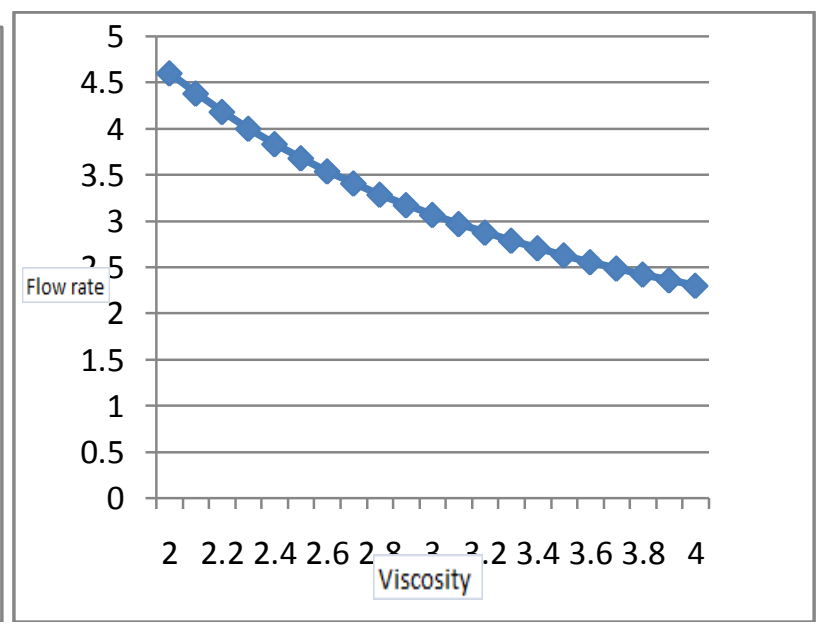

Fig.3

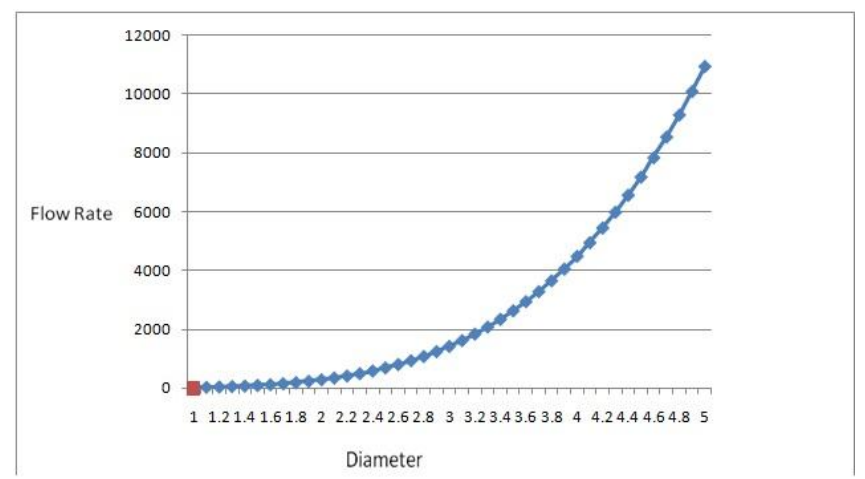

Fig.4

\section{Refrerences}

[1] Abby Jones, Oliver Pratt, "Physical Principles of Intra Arterial blood pressure measurement".Salford UK 2009.

[2] E Seidel, BM Eicke, "Reference values for vertebral Artery flow valume by duplex sonography in young and Elderly adults".American heart association 2012.

[3] Kenichi Takahata, Andrew dehennis "Stentenna : A microwmachined antenna stent for wireless monitoring of implantable microsensors" Ann Arbor,USA 2003.

[4] Andrew Dehennis and Kensall D.Wise "A double sided single chip wireless pressure sensor" The university of michigam 2002.

[5] Eun-Chul Park, jun-Bo Yoon "Hermetically sealed inductor-capacitor (LC) resonator for remote pressure monitoring" Japanese journal of applied physics 1998.

[6] William F Weitzel, Casey L Cotant, Zhijie Wen "Analysis of novel geometry-independent method for dialysis access pressureflow monitoring" USA 2008.

[7] J.W Trevan "The viscosity of blood" 